Document downloaded from:

http://hdl.handle.net/10251/99610

This paper must be cited as:

Arnal Pastor, MP.; Tallà-Ferrer, C.; Herrero-Herrero, M.; Martínez-Gómez Aldaraví, A.; Monleón Pradas, M.; Vallés Lluch, A. (2016). Scaffolds based on hyaluronan and carbon nanotubes gels. Journal of Biomaterials Applications. 31(4):534-543.

doi:10.1177/0885328216644535

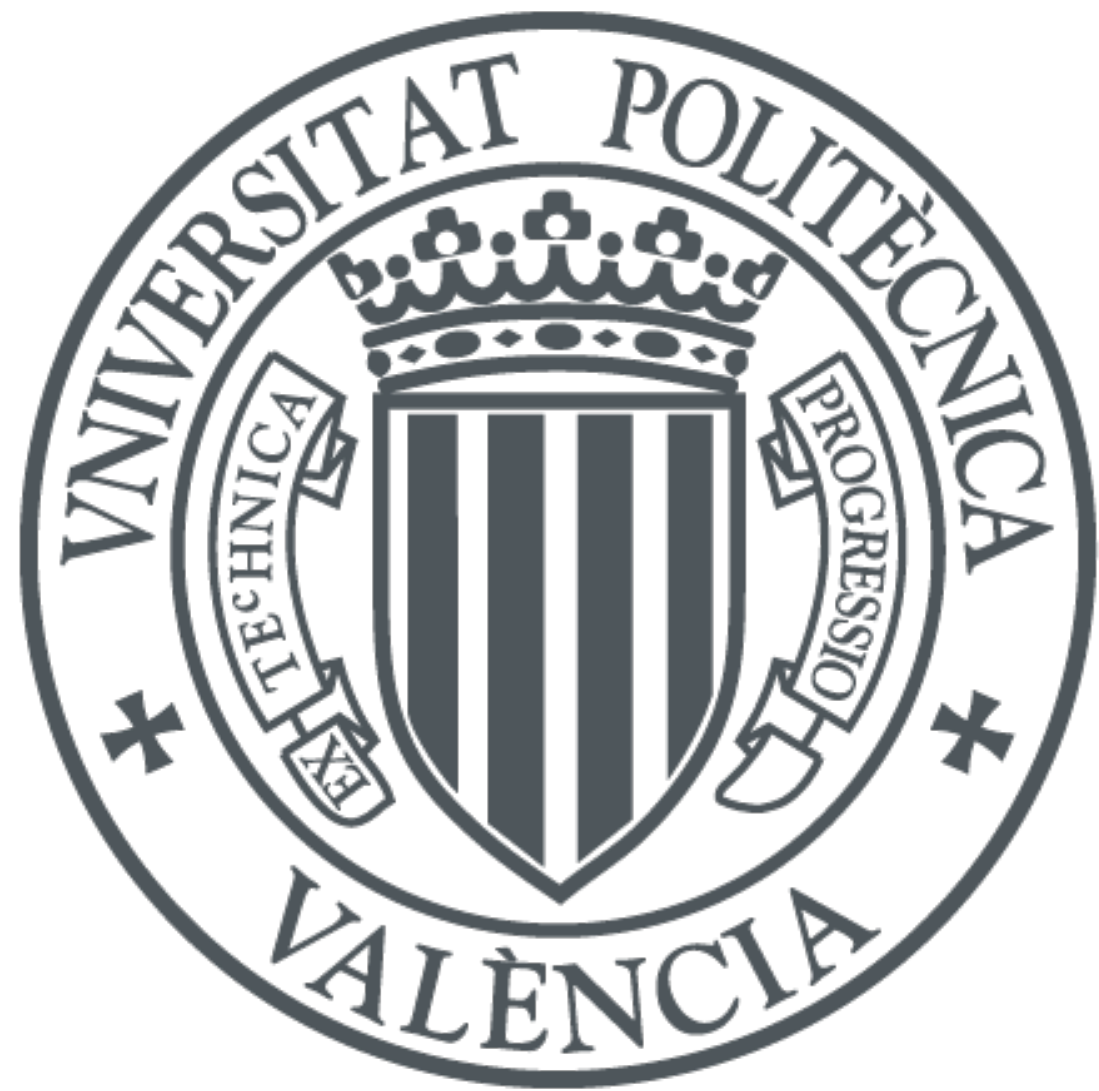

The final publication is available at

https://doi.org/10.1177/0885328216644535

Copyright SAGE Publications

Additional Information 


\section{SCAFFOLDS BASED ON HYALURONAN AND CARBON NANOTUBES GELS}

M. Arnal-Pastor ${ }^{1, \#}$, C. Tallà Ferrer ${ }^{1, \#}$, M. Herrero Herrero ${ }^{1, \#}$, A. Martínez-Gómez Aldaraví1, ${ }^{1,4}$ M. Monleón Pradas ${ }^{1,2}$, A. Vallés-Lluch ${ }^{1, *}$

${ }^{1}$ Centre for Biomaterials and Tissue Engineering, Universitat Politècnica de València, Spain

${ }^{2}$ Networking Research Center on Bioengineering, Biomaterials and Nanomedicine, Valencia, Spain

\# equal contribution

*corresponding author. Tel.: +3496387000; fax: +34963877276. E-mail: avalles@ter.upv.es

\section{ABSTRACT}

Physico-chemical and mechanical properties of hyaluronic acid (HA)/carbon nanotubes (CNTs) nanohybrids have been correlated with the proportion of inorganic nanophase and the preparation procedure. The mass fraction of $-\mathrm{COOH}$ functionalized CNTs was varied from 0 to 0.05 . Hyaluronic acid was crosslinked with divinyl sulfone (DVS) to improve its stability in aqueous media and allow its handling as a hydrogel. A series of samples was dried by lyophilization to obtain porous scaffolds whereas another was room dried allowing the collapse of the hybrid structures. The porosity of the former, together with the tighter packing of HA chains results in a lower water absorption and lower mechanical properties in the swollen state, because of the easier water diffusion. The presence of even a small amount of CNTs (mass fraction of 0.05) limits even more the swelling of the matrix, owing probably to hybrid interactions. These nanohybrids do not seem to degrade significantly during 14 days in water or enzymatic medium.

Keywords: hyaluronic acid; carbon nanotubes; nanohybrid; scaffold; lyophilization 


\section{Introduction}

Hyaluronic acid (HA) is one of the most commonly proposed hydrogels for tissue engineering, not only for being present in the organism but also because of its unique properties. HA is an abundant glycosaminoglycan distributed throughout the extracellular matrix (ECM) of many tissues and organs such as lungs, kidneys or brain [1]. HA has a structural function, but it also works as a signaling molecule, and takes part in the embryonic development and wound healing processes [2,3]. Furthermore, it plays a key role in the hydration balance and provides elasticity to the tissues because of its highly hygroscopic character [2], viscosity and high molecular weight. HA has been shown to also scavenge free radicals, to cause bacteriostasis, and to assist in tissue repair [4]. Moreover, low molecular weight HA has been found to be angiogenic [5]. HA is commonly used in medical applications, for example in ophthalmic viscosurgery, in osteoarthritic treatments, or as wrinkle filler [6]. Concerning applications in the tissue engineering field, HA hydrogels have been modified with laminin and tested in brain lesions [7], where they have shown to promote neurite extension. The combination of HA with collagen has also been studied with suitable results for brain tissue engineering [8,9]. Nonetheless, HA exhibits poor mechanical properties in its natural state, it is water-soluble, and thus it is cleared quickly [10]. To overcome these limitations, in tissue engineering strategies HA has been for example cross-linked with ethyl esters or benzyl esters [4], such as divinyl sulfone (DVS), showing good biocompatibility [11].

On the other hand, carbon nanotubes (CNTs) are graphene sheets rolled into continuous cylinders with diameters of $1 \mathrm{~nm}$ approximately [12]. CNTs exhibit exceptional electrical and mechanical properties, and are biostable [13]. Consequently, their incorporation into HA hydrogels is expected to improve their mechanical properties, 
stability and conductivity. In addition, the structure and dimensions of CNTs are similar to some elements of the neural system, such as ion channels, signaling proteins or elements of the neuronal cytoskeleton, being an advantage by enhancing molecular interactions and consequently yielding to a better control over physiological activity and neural information processing [14]. For these reasons, CNTs have been proposed for their use in neural regeneration. For instance, CNTs combined with collagen scaffolds [15] enhanced the levels of neuronal markers and neural grown factors expressed by mesenchymal cells. In [16], a nanohybrid consisting in chondroitin sulfate and CNTs induced the formation of neuronal networks.

Herein, CNTs functionalized with carboxylic groups (-COOH) have been used in combination with HA, due to their good dispersion in polar solvents [17] (better than that of non-functionalized CNTs), and their low cytotoxicity [18]. HA/CNTs nanohybrid scaffolds have here been obtained by freeze-drying (highly porous, due to the sublimation of water contained in the gels), with several proportions of CNTs. The spotlight of this work is set on the different role of CNTs in HA-based nanocomposites, attained by their processing methodology. Homologous nanohybrids obtained by solvent casting (bulk samples) have been produced as controls. Both series have been physico-chemically characterized. The effect of the production method and the fraction of incorporated nanotubes on the mechanical properties and stability in physiological conditions have been assessed.

These scaffolds are intended to be used to induce neural regeneration in tissues of the central or peripheral nervous system, on the one hand because HA resembles them in terms of mechanical properties $[19,20]$, and thus has been proposed for this purpose in several works [21-23], and on the other hand CNTs electrical conductivity makes them 
ideal for therapeutic applications at the neural tissue interface [24]. It is though beyond the scope of this paper to evaluate their biological development.

\section{Materials and methods}

\subsection{Preparation of lyophilized HA/CNTs (L) nanohybrids}

A 5 wt \% hyaluronic acid (HA; hyaluronic acid sodium salt, 1.63 MDa, obtained by fermentation of streptococcus equi. bacteria, 99\%, Sigma) solution was obtained by stirring in a $0.2 \mathrm{M}$ sodium hydroxide $(\mathrm{NaOH}$; extrapure, Scharlau) aqueous solution during 24 hours. To obtain the nanohybrids, different amounts of carbon nanotubes (MWCNT 95 wt\% 30-50 nm, 10-20 um long -COOH, Cheap Tubes Inc.) were previously dispersed in the $\mathrm{NaOH}$ solution by sonication (VWR USC300TH, Pennsylvania, USA) during 10 minutes at room temperature (RT) to avoid their aggregation. CNTs were added to the basic solutions to obtain a final mass fraction in the HA/CNT nanohybrid, $\omega_{C N T}$, of $0,0.5,1$ and $5 \mathrm{wt} \%$.

Next, divinyl sulfone (DVS; 97\%, Sigma Aldrich) was added to each hybrid solution as cross-linker at a ratio of 0.9 mol DVS per mol of HA monomeric units. After stirring for 10 seconds, 10 grams of each solution were poured into a $8.5 \mathrm{~cm}$-diameter Petri dish. The Petri dish was covered by a mesh fabric during 10 minutes, and next frozen overnight at $-20^{\circ} \mathrm{C}$. Then, the samples were lyophilized (Telstar Lyoquest -85 ) at $-80^{\circ} \mathrm{C}$ during 24 hours to create porous structures due to the sublimation of water. After demolding, the xerogels were swollen in water for 1 hour and punched into $5 \mathrm{~mm}$ diameter samples, next washed in a 50:50 vol\% water:acetone mixture for 2 days with a renewal at mid-time, followed by rinses in water for 3 days with renewals every 12 hours and lyophilized again to dry them. Samples were stored under vacuum protected 
from light until use. Hereafter, this series of samples will be referred to as $\mathrm{L} x$, where $\mathrm{L}$ means lyophilized and $x$ refers to the mass fraction of CNTs.

\subsection{Preparation of non- lyophilized HA/CNT (NL) nanohybrids}

An analogous procedure to that of the lyophilized hybrids series was followed. In this case, though, after the addition of DVS, the mixed solutions were allowed to crosslink in Petri dishes during 2 hours, covered with mesh fabrics. Next, they were frozen overnight at $-20^{\circ} \mathrm{C}$. Then, the samples were thawed to room temperature and swollen during one hour. Finally, the nanohybrids were punched into $5 \mathrm{~mm}$ diameter samples and the previously described washing procedure was followed. Samples were stored under vacuum protected from light until use. Hereafter, this series of samples will be referred to as NLx, where NL means non-lyophilized and $x$ refers to the mass fraction of CNTs.

\subsection{Scanning Electron Microscopy (SEM)}

The influence of CNTs at different mass fractions in the materials morphology was assessed in a JSM-5410 scanning electron microscope (SEM; JEOL. Ltd., Tokyo, Japan), at $15 \mathrm{~kW}$ of acceleration voltage and $15 \mathrm{~mm}$ of working distance. The samples were fractured in liquid nitrogen to obtain cross-section images and sputter-coated with gold under vacuum before observation.

\subsection{Determination of Equilibrium Water Content (EWC)}

Swelling of the nanohybrid materials was quantified at equilibrium, by weighing the dry samples, $m$, and after equilibration at $37^{\circ} \mathrm{C}$ in two different ambients: at $97 \%$ relative 
humidity (RH), and immersed in water. The first swelling medium was prepared with a potassium nitrate (Scharlab) supersaturated solution in a closed moisture chamber [25]. The samples were withdrawn from the moisture chamber after different times and immediately weighed, until no weight change was further observed; this value was taken for each nanohybrid as the equilibrium value, $m_{w}$. The equilibrium water content, $E W C$, was calculated as: $E W C=\frac{m_{w}-m}{m}$. Three replicates per composition were measured. A Mettler-Toledo XS105DU balance (Columbus, OH, USA) was used for this purpose.

\subsection{Determination of the density and porosity of the nanohybrid materials}

A Mettler AX205 (Mettler Toledo Inc., Columbus, OH, USA) balance, equipped with a Mettler ME 33360 density accessory kit was used to determine the density and porosity of dry samples and analogous series previously equilibrated in water. N-octane (Sigma Aldrich, 98\% purity, n-octane $=0.703 \mathrm{gcm}^{-3}$ ), which does not swell the nanohybrids, was used to make these quantifications. Three replicates per composition and conditioning were measured. The samples were weighed in air, $m$, after injection of noctane in their pores with the help of vacuum, $m_{i}$, and finally with their pores previously filled and the scaffold immersed in the liquid, $m_{l}$. The density, $\rho$, was calculated as the mass of the sample in air divided by the volume occupied by the nanohybrid, $V$, obtained by substracting the pores volume, $V_{\text {pores, }}$ from the apparent volume (geometrical volume of the sample), $V_{a p}$ :

$$
\rho=\frac{m}{V}=\frac{m}{V_{a p}-V_{\text {pores }}}=\frac{m}{\frac{m_{i}-m_{l}}{\rho_{l}}-\frac{m_{i}-m}{\rho_{l}}}=\frac{m}{m-m_{l}} \rho_{l}
$$

The porosity, $\pi$, was next obtained as the pores volume fraction: 


$$
\pi=\frac{V_{\text {pores }}}{V_{a p}}=\frac{m_{i}-m}{m_{i}-m_{l}}
$$

\subsection{Compressive tests}

Compressive mechanical tests were performed in a Seiko TMA/SS6600 dilatometer (Seiko Instruments Inc, Chiba, Japan) in water at $37^{\circ} \mathrm{C}$, with the samples previously swollen in water, to simulate physiological conditions. A steel rod of $3 \mathrm{~mm}$ of diameter was used for this purpose. Five replicates per composition were tested, from 0 to 1500 $\mathrm{mN}$ at $100 \mathrm{mN} / \mathrm{min}$. The compressive elastic modulus, $E$, was obtained as the slope of the stress-strain curves in the initial linear region.

\subsection{Degradation/stability assays}

In order to investigate the enzymatic degradation process of HA, as well as the effect of CNTs on it, HA lyophilized (L) and non-lyophilized samples (NL) and nanohybrids with 0.5 wt \% in carbon nanotubes (L0.5 and NL0.5) were subjected to degradation, 8 replicates per time point. For that purpose, the samples were immersed in an aqueous solution of bovine testicular hyaluronidase (BTH, Type IV-S, 750-3000 units/mg, Sigma-Aldrich) $10 \mathrm{u} / \mathrm{ml}$ at $37^{\circ} \mathrm{C}$, for $24,72,168$ and 336 hours. The solution was not changed throughout the experiment.

HA degrades naturally in human body by hyaluronidase enzymes and reactive oxygen species (ROS) [26]. As degradation products, hexa and tetrasaccharides are mainly obtained. The BTH enzyme was selected for having the same action mechanism as human hyaluronidase and for having been used in other works [27-30]. The concentration used mimics that of physiological environment. 
Degradation assays are usually carried out in PBS (ionic strength of $0.165 \mathrm{M}$ ) because at its ionic strength the enzyme activity is optimized [31,32]. However, distilled water was used here in its place to dissolve the enzyme as a compromise choice, to avoid the presence of salts that gives false positives in terms of weight loss.

In parallel, stability in mili-Q water was assessed by introducing a second set of samples in the same volume ( $2 \mathrm{ml} / \mathrm{sample}$ ) of water at $37^{\circ} \mathrm{C}$ (non-enzymatic degradation). Samples were conditioned in water before any assay. After withdrawal, the enzyme in each vial was thermally inactivated at $100^{\circ} \mathrm{C}$ for 90 seconds (the same procedure was applied to those samples immersed in a non-enzymatic medium, for comparison) and vials were stored at $4-8^{\circ} \mathrm{C}$ until use.

The variation of the materials properties over time was evaluated by mass loss determination, morphology observation under SEM and compressive tests; for the first two experiments, samples were previously lyophilized, whereas for the latter, samples

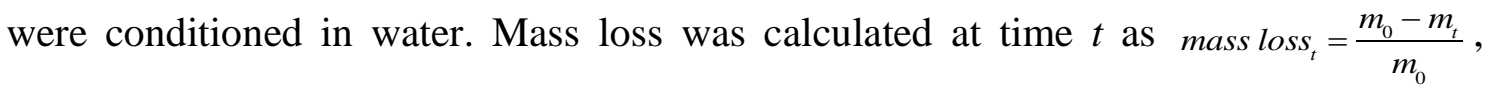
where $m_{0}$ and $m_{t}$ were the initial and at time $t$ weight of the sample, respectively.

\subsection{Statistics}

All the experimental data are given as mean \pm standard deviation. Statistical assessment of significant variance was performed through a one-way ANOVA with the Statgraphics Centurion XVI.II software (Statistical Graphics Corp., Princeton, NJ). Statistical tests were performed at 95\% significance level (p-value $<0.05$ ). Differences are statistically significant unless otherwise stated. 


\section{Results and discussion}

\subsection{Morphology of the nanohybrids}

The samples acquired an increasingly darker blackish tone (Figure 1) as the fraction of CNTs increased. After drying, the gels became rigid (vitreous), and pure HA, which was transparent as a gel, became white.

Figure 1 also shows the surface and section SEM images of the lyophilized HA/CNTs nanohybrids series. The L-samples show a microporous honeycomb structure, attributed to the formation of water crystals upon freezing and their subsequent sublimation during the lyophilization step. On the L0 surface and transversal section (images A and E), uniform pore sizes were observed, with diameter of 152.1 $222.1 \mu \mathrm{m}$. Evidence of CNTs aggregates was not found in any case; in all nanohybrids an interconnected honeycombtype porous structure (typical of lyophilized hydrogels) was observed. This structure, though, seems to be slightly modified by the presence of CNTs: the average size of the pores increases up to around $340 \mu \mathrm{m}$ and their number decreases, especially in the

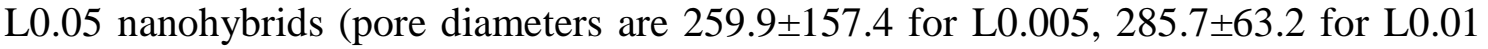
and $341.5 \pm 46.1$ for L0.05). This could be due to the difficulty in obtaining completely homogeneous aqueous solutions with this CNTs fraction, and partial phase separation, yielding to larger water domains that sublimate resulting in pores. These results are in good agreement with those obtained in [33] where a low CNTs concentration (2 wt\%) opens the structure giving rise to large pores.

As for the non-lyophilized samples (images I-P), the SEM micrographies show nonporous surfaces, wrinkled and folded due to the collapse of highly hydrated structures. For the highest concentration of CNTs, randomly dispersed bulges with an average size 
of $30 \mu \mathrm{m}$ were observed (highlighted by a dotted line), which might be aggregations of CNTs. A similar finding was reported in [34]: HA nanohybrids with mass fractions of silica up to 0.05 display a particulate-HA matrix morphology, with silica aggregates more or less homogeneously distributed throughout the organic matrix, that progressively disappear at higher silica mass fractions to give a fine continuous interpenetrated silica network.

The dependence of the density of L and NL nanohybrids on the mass fraction of CNTs is shown in Figure 2 (A). This Figure sheds some light on the interaction between HA and CNTs at varying concentrations. In the dry state, the density of L samples was expected to be higher than those of NL samples, according to the hypothesis that lyophilization yields a denser HA network, with intra and intermolecular interactions, and pores of larger sizes. However, the density was somewhat lower at low CNTs concentrations (up to 1\%) and non-statistically significant differences could be found. This could be attributed to the discrepancy in the crosslinking times: NL samples were allowed to crosslink for $2 \mathrm{~h}$ instead of $10 \mathrm{~min}$ and it seems that at low $\omega_{C N T}$ the crosslinking through DVS that occurs as the solution dries during this extra time is more effective than the hydrogen bonds between HA units (inter and intramolecular ones) promoted by lyophilization. This does not happen at higher $\omega_{C N T}$, because CNTs impede such drying and molecules are less likely to meet and crosslink, and so the $\mathrm{L}$ homologous hybrids get denser. Lyophilization allows a more effective elimination of water and vitrification of $\mathrm{HA}$, and are thus the data for the $\mathrm{L}$ samples those that reveal the effect of CNTs increasing its density.

These results can be compared with those obtained in [34] for non-lyophilized HA hybrids with $\mathrm{SiO}_{2}$ nanoparticles, where the density of $\mathrm{HA}$ increased from $1.43 \mathrm{~g} / \mathrm{ml}$ to $1.52 \mathrm{~g} / \mathrm{ml}$ with $5 \% \mathrm{SiO}_{2}$. Herein, the density increases provided that samples are dried 
by lyophilization, but not the other way. Maybe the drying process, which consisted there in a long curing not disturbed by freezing, is behind such tighter packing.

The scaffolds' porosity is a key parameter in determining their suitability for the intended application, as it affects different processes such as nutrients diffusion, vascularization or cells colonization. The porosity of dry and swollen samples is presented in Figure 2B and C. It is high for lyophilized materials ( 95\%) when dry, and decreases as the samples swell, mainly for those with low $\omega_{C N T}$, which have less impediments to swell or those with high $\omega_{C N T}(0.05)$, which lodge more water because of greater availability of -COOH groups from CNTs. Upon swelling, the diameter of pores is reduced by water opening and stretching the polymeric network. For nonlyophilized ones, the porosity does not reach $0(\sim 20 \%)$ in either state, probably due to the folds observed in the SEM micrographies (Figure 1 I-P), which play as voids and hold water.

\subsection{Modulation of HA swelling by the inclusion of CNTs}

The influence of CNTs on the HA-network's capacity to swell was followed by equilibrating the weight of nanohybrids immersed in liquid water and in a water vapor ambient of $97 \%$ relative humidity. Figure 3 represents the equilibrium water content (EWC) of the samples swollen in both media. To better analyze the effect of the inclusion of inorganic particles, the incorporated water was also referred to the HA mass (in place of the nanohybrid mass), assuming that only HA contributed to the sorption, independently of the CNTs content, $E W C^{\prime}=\frac{m_{w}-m}{m_{H A}}=\frac{E W C}{\omega_{H A}}, \omega_{H A}$ being the HA mass fraction in each nanohybrid. The values obtained can also be compared with an ideal $E W C$ calculated as $E W C^{i d}=E W C_{H A} \cdot \omega_{H A}$, where $E W C_{H A}$ is the $E W C$ of pure HA. 
As expected, $E W C$ in a vapor ambient was much lower (0.1 to 0.55 times its dry weight depending on the CNTs mass fraction) than in liquid water (35 to 55-fold) because only water molecules establishing hydrogen bonds with the polymer chains are computed in the former, whereas in the latter large volumes of free water can also be lodged in the HA matrix as it expands, in addition to the water lodged in the scaffold's pores of lyophilized samples. Water can indeed be found in hydrogels as one of three types: bound water, i.e., that ascribed to those water molecules strongly interacting through hydrophilic groups of the polymer, free water, which refers to bulk domains lodged in the gel network (and in large pores obtained by lyophilization) that do not take part in hydrogen bonds with polymer molecules, and semi-bound water, with intermediate properties between those of bound and free water [35]. Free water can be found when samples are immersed in water but not equilibrated in a humid atmosphere.

As for the drying effect, lyophilized materials swelled less than non-lyophilized ones in a humid ambient (B and D). This result was expected since freeze-drying packs the HA polymer chains, favoring irreversible hydrogen bonds between them, thus hindering the water diffusion through the material. In contrast, the absence of such extra interactions in non-lyophilized materials allows the easier diffusion of water, and hence a greater EWC. As far as the role of CNTs is concerned, for 97\% RH one can compare their influence on the EWC depending on the drying process: the presence of even a small fraction of CNTs elicits a decrease on the swelling capacity. The EWC' values changed with the CNTs fraction similarly as EWC, being always slightly above them, but below the $E W C^{\text {id }}$ linear behavior. This trend suggests that the presence of CNTs within the polymeric network modifies the hydrophilicity of HA molecules: even at very small amounts they constrain the swelling of the organic network. This regulation is though more pronounced in non-lyophilized materials: in lyophilized ones HA chains are 
packed in the drying process itself that leaves less polar groups exposed, whereas in non-lyophilized ones, this regulation plausibly occurs as long as CNTs carboxyl groups interact with HA chains through hydrogen bonds.

When samples were immersed in liquid water those non-lyophilized showed, though, a different pattern: the inclusion of CNTs enabled the materials to swell more. This effect was previously observed in [34] for non-lyophilized nanohybrids with $\mathrm{SiO}_{2}$ particles, and was attributed to a changing hydrophilicity of the HA network when in interaction with inorganic 'defects', together with a plausible contribution of the inorganic species to the overall hydrophilicity. Lyophilization forces the packaging and interaction of HA chains in spite of the presence of CNTs, which will end up entrapped, so both will be less available to interact with water and differences between samples are not herein statistically significant.

\subsection{Improvement of mechanical properties}

Figure 4 ( $\mathrm{A}$ and $\mathrm{B}$ ) shows the stress-strain curves from the compression tests of some samples as examples and the elastic modulus obtained from the initial linear region of such curves for each CNTs content and treatment. For lyophilized plasticized (equilibrated in water) samples, an elastic modulus around $30 \mathrm{kPa}$ was obtained and no influence of the CNTs was observed. Nonetheless, non-lyophilized materials reached higher values, between 135 and $235 \mathrm{kPa}$. These differences are due to the porous structure of the L-samples, so they have less water retained within the matrix than NL ones. Moreover, the porous structure allows the water extrusion more easily through the pores than from the bulk material of the NL-samples, i.e., because of its incompressibility, water retained in the material plays an important role on the resulting elastic modulus. 
In contrast, the presence of CNTs seemed to slightly affect the elastic modulus in NLsamples, probably due to the fact that CNTs, disconnected at low concentrations, are only able to reinforce the polymeric network once their concentration is high enough. To better analyze the effect of entrapped water on the mechanical properties, the elastic modulus of the samples is depicted in Figure 4C against their EWC, which had been quantified before the compression tests. L-samples did not show a significant variation of the elastic modulus with $E W C$, probably due to the easy extrusion of water from their pores, as mentioned before. The porosity is the parameter that governs the mechanical properties of these scaffolds. On the contrary, in NL-samples, the higher EWC the greater their elastic modulus; this is because of poroelasticity [36,37], which arises from the combination of the incompressibility of water and its hindered extrusion from the gelly matrix. In this series, the role of CNTs is linked to that of water: at low concentrations they hinder the expansion and swelling of the HA matrix and thus reinforce it, and at higher mass fractions they contribute to swelling and it is water which improves indeed mechanical properties.

\subsection{Improvement of stability in aqueous media}

Macroscopically, after two weeks, samples submitted to degradation tests exhibited the same appearance and consistence as non-degraded ones. Nonetheless, holes of some microns were observed for the hyaluronidase-degraded samples under SEM (Figure 5); these holes were more abundant in pure samples than in those with $0.5 \%$ CNTs, and their number and size increased with time. NL-samples did not show any evidence of degradation (Sup. Figure 1). 
In order to better analyze the evolution of properties with the degradation time, the weight loss (Figure 6) was also quantified. Lyophilized materials slightly lost more weight than non-lyophilized ones: for the lyophilized series the mass loss reached $5 \%$ in bare samples and $4 \%$ in those with $0.05 \%$ CNTs, whereas the non-lyophilized homologous samples had losses of $1.7 \%$ and $1.6 \%$, respectively. This difference could be attributed to the hindered diffusion of HA fragments from the gelly non-lyophilized samples, in comparison with that from scaffolds with sizable pores. Weight loss occurs mainly during the first 150 days, and smoothes later on. Carbon nanotubes seem to confer more stability to the lyophilized samples against degradation; results are, though, not statistically significant. Non-enzymatic degradation did not result in a significant weight loss during the 300 days of experiment.

The elastic moduli of the samples did not show significant changes with the degradation time up to 14 days; they kept the initial values independently of their drying process. The presence of hyaluronidase in the medium did not affect significantly, either, the mechanical properties of the samples (Sup. Figure 2).

\section{Conclusions}

The two-steps freeze-drying procedure followed herein, with a swelling in water and an acetone/water rinsing in between, allowed the fabrication of porous materials based on HA, with CNTs mass fractions up to 0.05. These scaffolds showed a highly porous network with interconnected pores of 100 to $300 \mu \mathrm{m}$ in diameter, suitable for tissue engineering applications. Lyophilization itself forces the packing and interaction of HA molecules, modulating their swelling. The presence of even a small amount of CNTs acts in the same direction, leading to equilibrium water contents between 35 and 55 
fold. The porosity governs the mechanical properties of these scaffolds (135-235 kPa), whereas in non-lyophilized nanohybrids the water sorption, regulated by CNTs, explains the higher elastic moduli because of a poroelasticity effect. None of the samples showed a significant degradation after 336 hours, either in water or hyaluronidase-containing medium. 


\section{References}

[1] Fraser, J. E., Laurent, T. C., Laurent, U. G. (1997). Hyaluronan: its nature, distribution, functions and turnover. J Intern Med, 242, 27-33.

[2] Day, M., Macarenhas, M. (2004). Signal transduction associated with Hyaluronan. In H. Garg, C. Iales, Chemistry and Biology of Hyaluronan (pp. 153-188). Elsiever Ltd.

[3] Vasi, A., Ionel Popa, M., Butnaru, M., Dodi, G., Verestiuc, L. (2014). Chemical functionalization of hyaluronic acid for drug delivery applications. Mater Sci Eng C, 38, 177-185.

[4] Ulery, B. D., Nair, L. S., Laurecin, C. T. (2011). Biomedical Applications of Biodegradable Polymers. Polym Phys, 49, 832-864.

[5] Gaffney, J., Matou-Nasri, S., Grau-Olivares, M., Slevin, M. (2010). Therapeutic applications of hyaluronan. Mol Biosystems, 6(3) , 437-443.

[6] Robert, L. (2014). Hyaluronan, a truly “youthful” polysaccharide. Its medical applications. Pathologie Biologie, 63(1), 32-34.

[7] Hou, S., Xu, Q., Tian, W., Cui, F., Cai, Q., Jun , M., Lee, I.-S. (2005). The repair of brain lesion by implantation of hyaluronic acid hydrogels modified with laminin. J Neurosci Meth, 148, 60-70.

[8] Wang, T.W., Spector, M. (2009). Development of hyaluronic acid-based scaffolds for brain tissue engineering. Acta Biomater, 5, 2371-2384.

[9] Wei Y. T., Tian W. M., Yu X., Cui F. Z., Hou S. P., Xu Q.Y., Lee I.-S. (2007) Hyaluronic acid hydrogels with IKVAV peptides for tissue repair and axonal regeneration in an injured rat brain. Biomed Mater, 2, S142-S146.

[10] Kablik, J., Monheit, G.D., Yu, L., Chang, G., Gershkovich, J. (2009). Comparative physical properties of hyaluronic acid dermal fillers. Dermatol Surg, 35(s1) , 302-312.

[11] Iannitti, T., Bingol, A., Rottigni, V., Palmeri, B. (2013). A new highly viscoelastic hyaluronic acid gel: rheological properties, biocompatibility and clinical investigation in esthetic and restorative surgery. Int J Pharm, 456, 583-592.

[12] Rafique, M.M.A., \& Iqbal, J. (2011). Production of carbon nanotubes by different routes-a review. Journal of encapsulation and adsorption sciences, 1(02) , 29.

[13] Fraczek-Szczypta, A. (2014). Carbon nanomaterials for nerve tissue stimulation and regeneration. Mater Sci Eng C, 34, 35-49. 
[14] Nunes, A., Al-Jamal, K., Nakajima, T., Hariz, M., Kostarelos, K. (2012). Application of carbon nanotubes in neurology: clinical perspectives and toxicological risks. Arch Toxicol, 86(7) , 1009-1020.

[15] Lee, J.H., Lee, J.Y., Yang, S.H., Lee, E.J., Kim, H.W. (2014). Carbon nanotubecollagen three-dimensional culture of mesenchymal stem cells promotes expression of neural phenotypes and secretion of neurotrophic factors. Acta Biomater, 10(10), 4425-4436.

[16] Serrano, M. C., Nardecchia, S., García-Rama, C., Ferrer, M. L., Collazos-Castro, J. E., del Monte, F., Gutiérrez, M. C. (2014). Chondroitin sulphate-based 3D scaffolds containing MWCNTs for nervous tissue repair. Biomaterials, 35, 1543-1551.

[17] Lee, J., Kim, M., Hong, C.K., Shim, S.E. (2007). Measurement of the dispersion stability of pristine and surface-modified multiwalled carbon nanotubes in various nonpolar and polar solvents. Meas Sci Technol, 18(12), 3707.

[18] Zhang, T., Tang, M., Kong, L., Li, H., Zhang, T., Zhang, S., Pu, Y. (2012). Comparison of cytotoxic and inflammatory responses of pristine and functionalized multi-walled carbon nanotubes in RAW 264.7 mouse macrophages. J Hard Mater, 219-220, 203-212.

[19] Seidlits, S.K., Khaing, Z.Z., Petersen, R.R., Nickels, J.D., Vanscoy, J.E., Shear, J.B. Schmidt, T.E. (2010). The effects of hyaluronic acid hydrogels with tunable mechanical properties on neural progenitor cell differentiation. Biomaterials, 31 3930-3940.

[20] Lam, J., Truong, N.F., Segura, T. (2013). Design of cell-matrix interactions in hyaluronic acid hydrogel. Acta Biomater, 10, 1571-1580.

[21] Tian, W.M., Hou, S.P., Ma, J., Zhang, C.L., Xu, Q.Y., Lee, I.S., Li, H.D., Spector, M., Cui, F.Z. (2005). Hyaluronic acid poly-D-lysine-based three-dimensional hydrogel for traumatic brain injury. Tissue Eng, 11, 513-525.

[22] Wang, T., Spector, M. (2009). Development of hyaluronic acid-based scaffolds for brain tissue engineering. Acta Biomater, 5, 2371-2384.

[23] Liang, Y., Walczak, P., Bulte, J.W. (2013). The survival of engrafted neural stem cells within hyaluronic acid hydrogels. Biomaterials, 34, 5521-5529.

[24] Bosi, S., Fabbro. A., Ballerini, L., Prato, M. (2013). Carbon nanotubes: a promise for nerve tissue engineering? Nanotechnol Rev, 2, 47-57.

[25] Greenspan, L. (1977). Humidity fixed points of binary satured aqueous solutions. $J$ Res Nat BurStan A Phys Chem, 81A(1), 89-96. 
[26] Schanté, C., Zuber, G., Herlin, C., Vandamme, T. (2011). Chemical modifications of hyaluronic acid for the synthesis of derivatives for a broad range of biomedical applications. Carbohyd Polym, 85, 469-489.

[27] Burdick, J.A., Chung, C., Jia, X., Randolph, M.A., Langer, R. (2005). Controlled degradation and mechanical behavior of photopolymerized hyaluronic acid networks. Biomacromolecules, 6,386-91.

[28] Astériou, T., Vincent, J.C., Tranchepain, F., Deschrevel, B. (2006). Inhibition of hyaluronan hydrolysis catalysed by hyaluronidase at high substrate concentration and low ionic strength. Matrix Biol, 25(3),166-74.

[29] Deschrevel, B., Tranchepain, F., Vincent, J.C. (2008). Chain-length dependence of the kinetics of the hyaluronan hydrolysis catalyzed by bovine testicular hyaluronidase. Matrix Biology, 27, 475-486.

[30] La Gatta, A., Schiraldi, C., Papa, A. De Rosa, M. (2011). Comparative analysis of commercial dermal fillers based on crosslinked hyaluronan: Physical characterization and in vitro enzymatic degradation. Polym Degrad Stab, 96, 630-636.

[31] Alburn, H.E., Whitley, R.W. (1951). Factors affecting the assay of hyaluronidase. $J$ Biol Chem, 192, 379-393.

[32] Bachtold, J.G., Gebhardt, L.P. (1952). The determination of hyaluronidase activity as derived from its reaction kinetics. J Biol Chem, 194, 635-639.

[33] Bhattacharyya, S., Guillot, S., Dabboue, H., Tranchant, J.F., Salvetat, J.P. (2008). Carbon nanotubes as structural nanofibers for hyaluronic acid hydrogel scaffolds. Biomacromolecules, 9(2), 505-509.

[34] Vallés-Lluch, A., Poveda-Reyes, S., Amorós, P., Beltrán, D., Monleón-Pradas, M. (2013). Hyaluronic acid-silica nanohybrid gels. Biomacromolecules, 14, 42174225.

[35] Omidian, H., Park, K. (2010). Introduction to Hydrogels. In Biomedical Applications of Hydrogels Handbook; Ottenbrite, R.M., Ed.; Springer: London, UK. pp. 1-16.

[36] Detournay, E.; Cheng, A.H.-D. Fundamentals of poroelasticity. Comprehensive Rock Engineering: Principles, Practice and Projects, Vol. II, Analysis and Design Method; Pergamon Press: New York, 1993; pp 113-171.

[37] Fornells, P.; García-Aznar, J. M.; Doblaré, M. (2007) A finite element dual porosity approach to model deformation-induced fluid flow in cortical bone. Ann Biomed Eng, 35, 1687-1698. 


\section{Figures}

Figure 1. Macroscopic image showing the nanohybrids with different CNTs mass fraction (above row) and SEM images of the surface (A-D; I-L) and section (E-H; M-P) of the lyophilized (A-H) and non-lyophilized (I-P) series. Scale bar: $300 \mu \mathrm{m}$.

$\omega$ CNTs

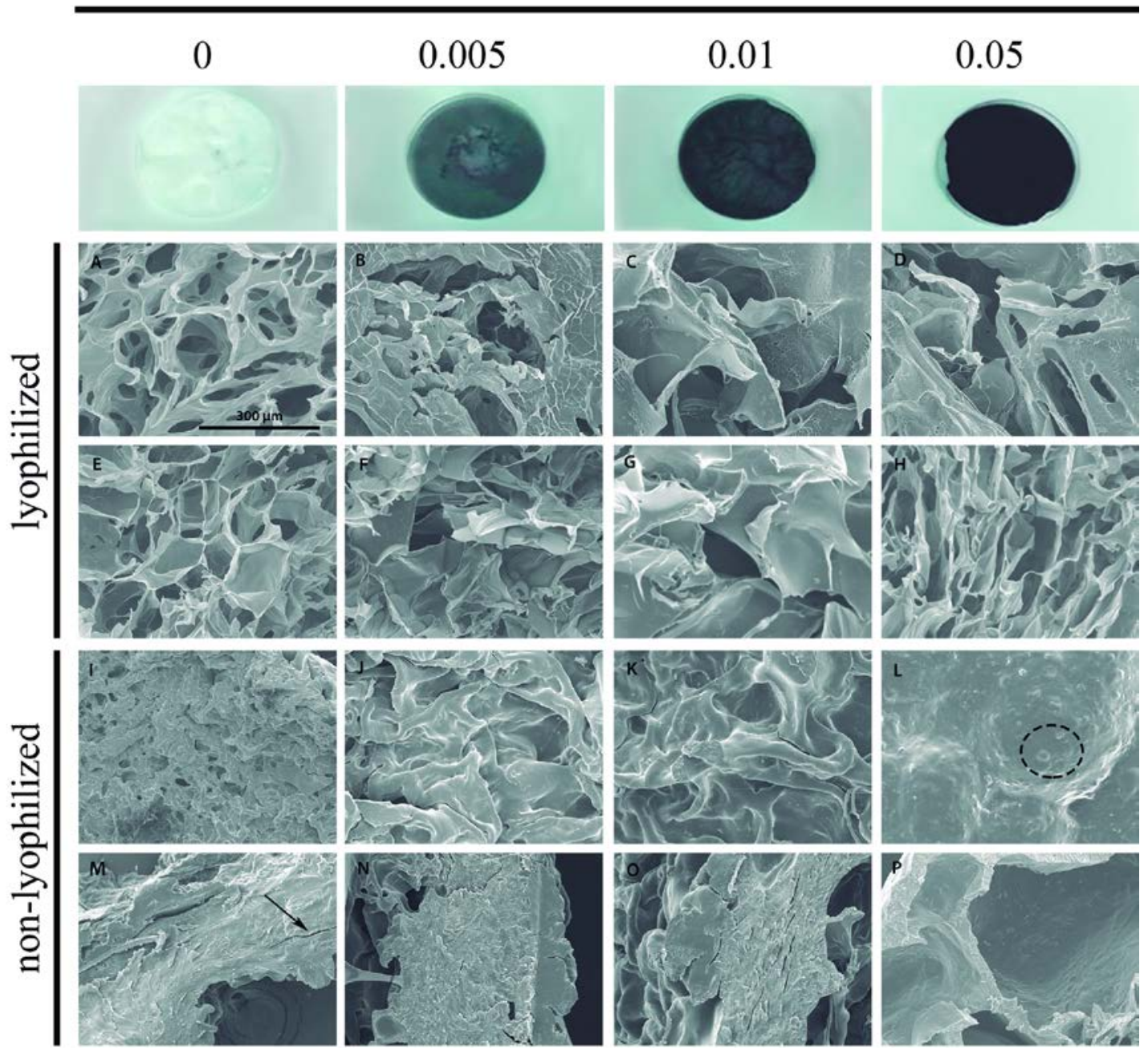


Figure 2. Density of lyophilized and non-lyophilized nanohybrids (A), and porosity of dry (B) and swollen (97\% RH atmosphere) samples (C) as a function of the CNTs mass fraction. Differences in densities (A) are not statistically significant neither with the drying process nor with the composition of the samples. Variations in (B) attributed to the CNTs variation for samples dried likewise are not statistically significant.

A)

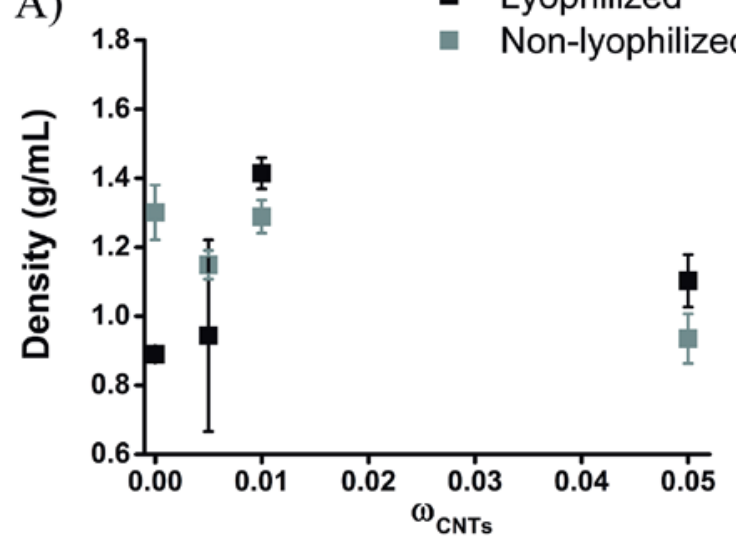

B)
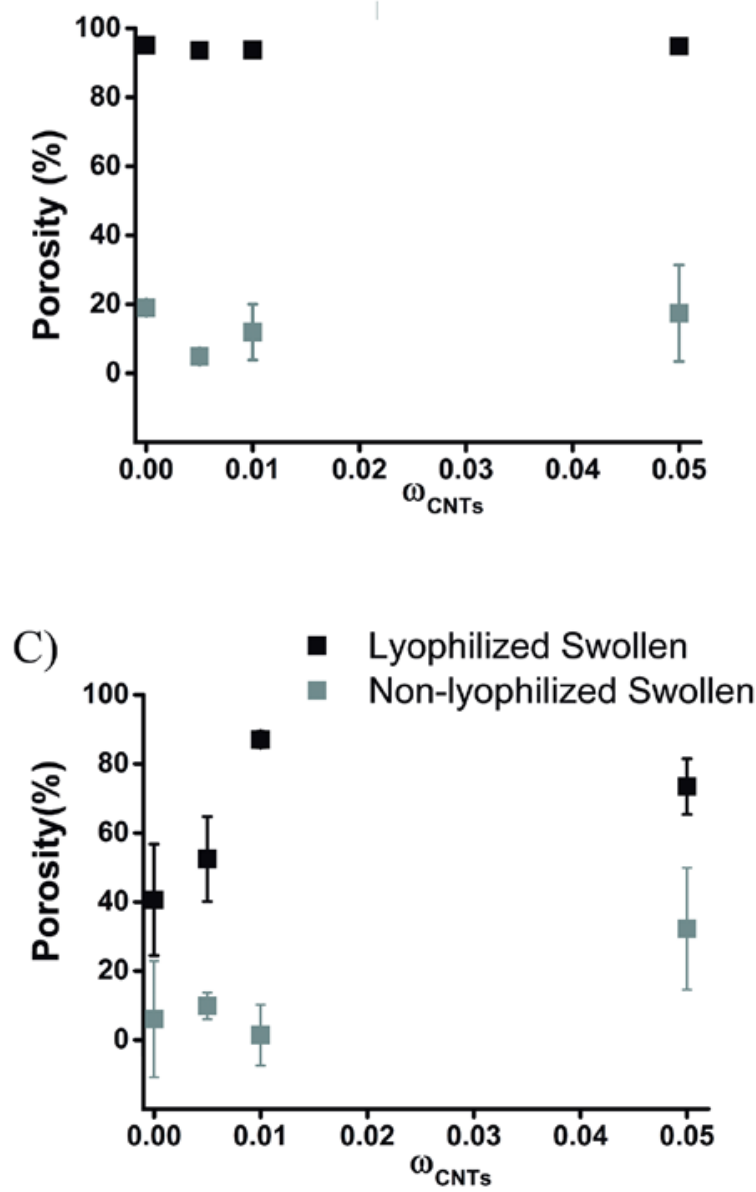
Figure 3. Water content after equilibration $(E W C)$ of L- (A, B) and NL-samples (C, D) in liquid water (A, C) and in a $97 \% \mathrm{RH}$ vapor ambient $(\mathrm{B}, \mathrm{D})$, as a function of the CNTs mass fraction content: referred to the dry mass of each nanohybrid (EWC) and to the HA mass in it (EWC'), and predicted from the $E W C$ of $\mathrm{HA}\left(E W C_{\mathrm{id}}\right)$. Differences between L- samples with different CNTs mass fractions are not statistically significant when swollen in water (A).

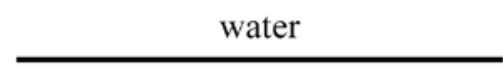

A)

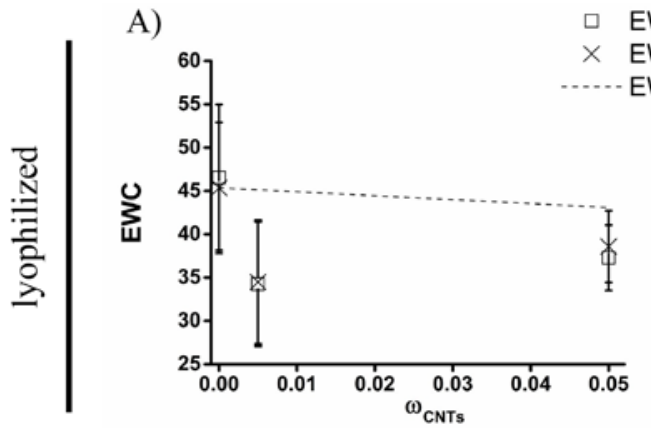

C)

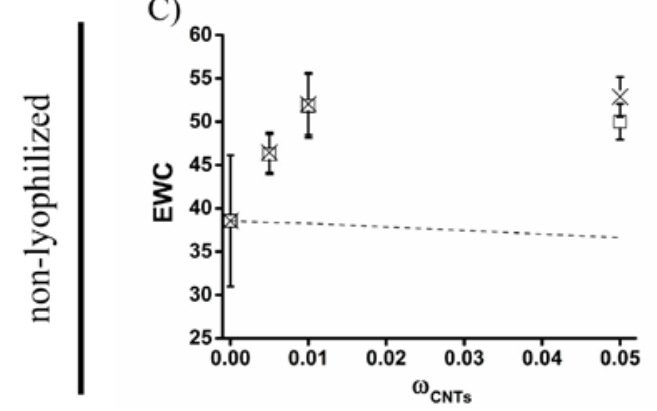

B)

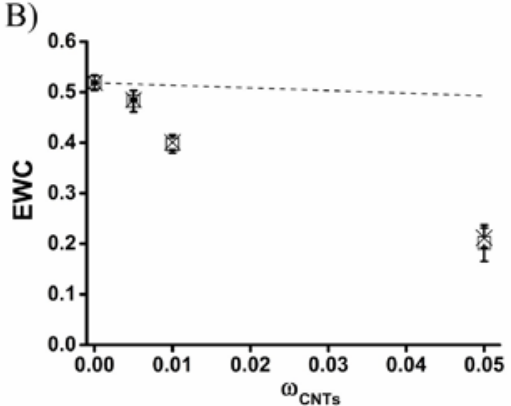

D)

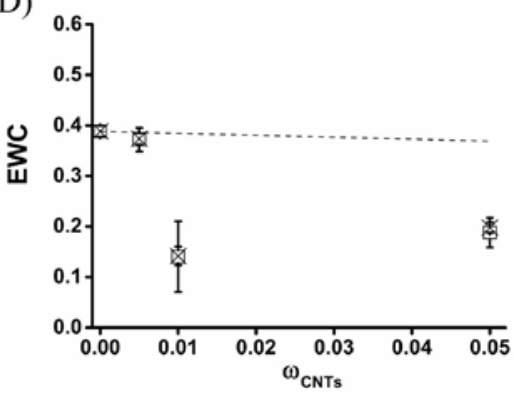


Figure 4. Stress-strain curves for L0, L0.05, NL0 and NL0.05 as example, showing the zone where data were fitted to a linear regression to get the elastic moduli (A). Compressive elastic moduli of lyophilized and non-lyophilized samples as a function of the CNTs mass fraction (B) and EWC (C). Differences due to variations in the CNTs mass fraction are not statistically significant.
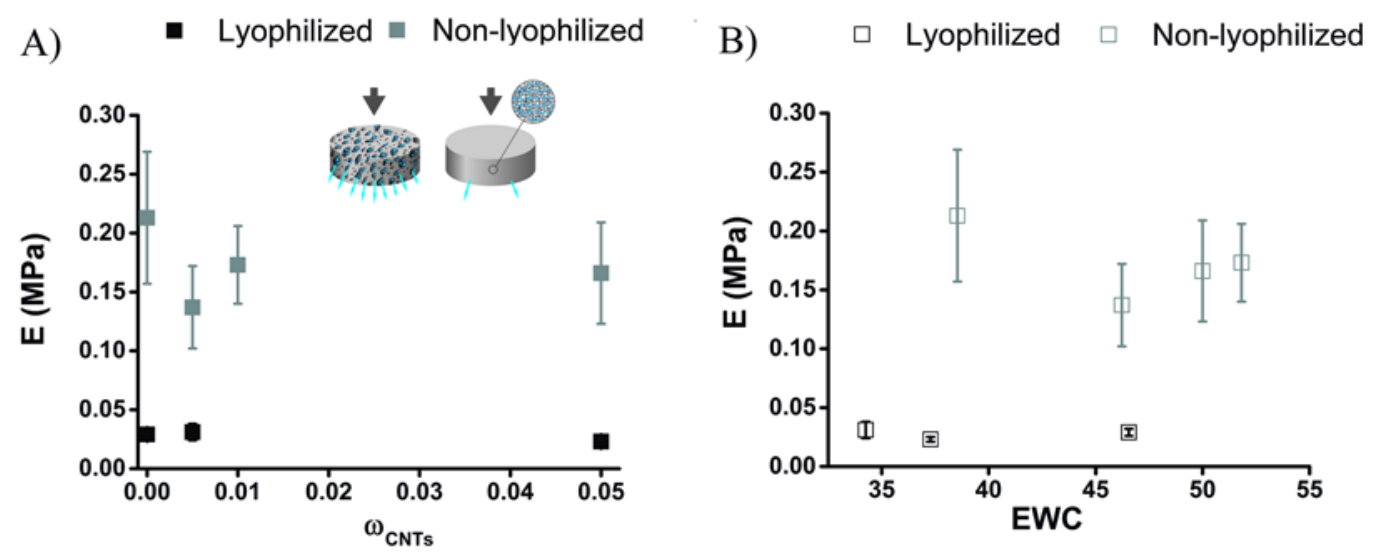

Figure 5. SEM surface images of HA-based lyophilized materials (A-D) without CNTs and $(\mathrm{E}-\mathrm{H})$ with a CNTs mass fraction of 0.005, degraded in water during 168 (A, E) and 336 hours $(\mathrm{B}, \mathrm{F})$, and in the presence of hyaluronidase during $168(\mathrm{C}, \mathrm{G})$ and 336 hours (D, H). The dotted circles show possible areas of hydrolysis.
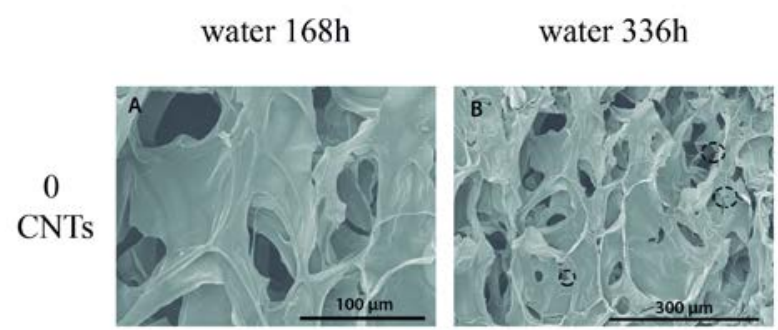

hyal $168 \mathrm{~h}$

hyal $336 \mathrm{~h}$
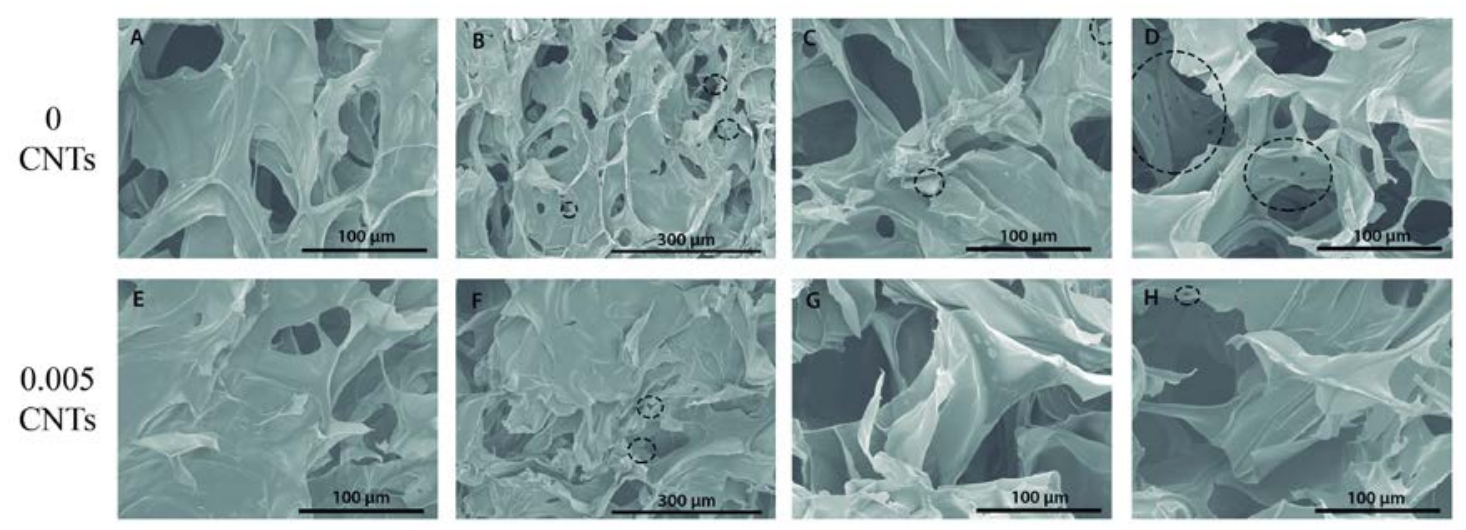
Figure 6. Weight loss of lyophilized (A) and non-lyophilized (B) bare samples and nanohybrids with $0.05 \%$ CNTs in medium containing hyaluronidase. Pure HA samples degraded non-enzymatically are used as control. Non-statistically significant differences were found between samples prepared following the same drying procedure.

A)

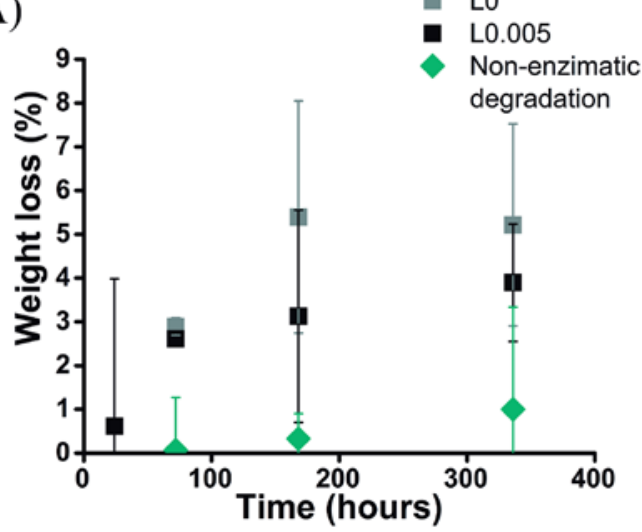

B)

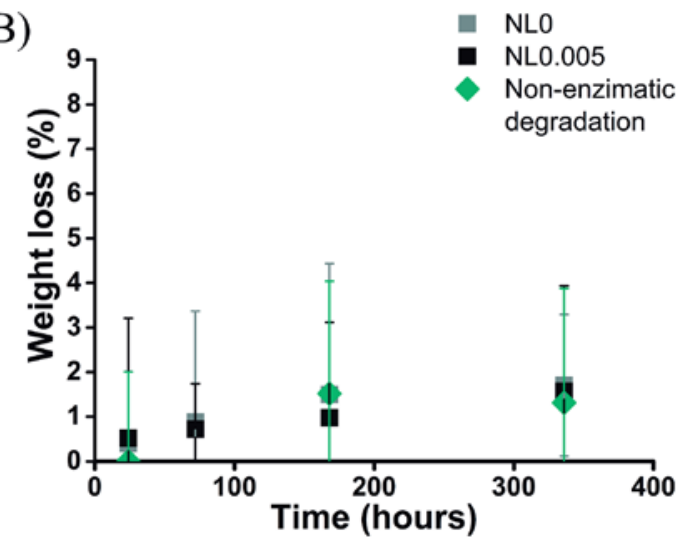

Sup. Figure 1. SEM surface images of HA-based non-lyophilized materials (A-C) without CNTs and (D-F) with a CNTs mass fraction of 0.005, degraded in water during 168 (A, D), and in the presence of hyaluronidase during 168 (B, E) and 336 hours (C, F).

water $168 \mathrm{~h}$

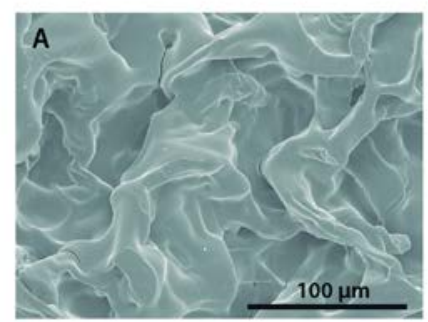

0.005

CNTs hyal $168 \mathrm{~h}$
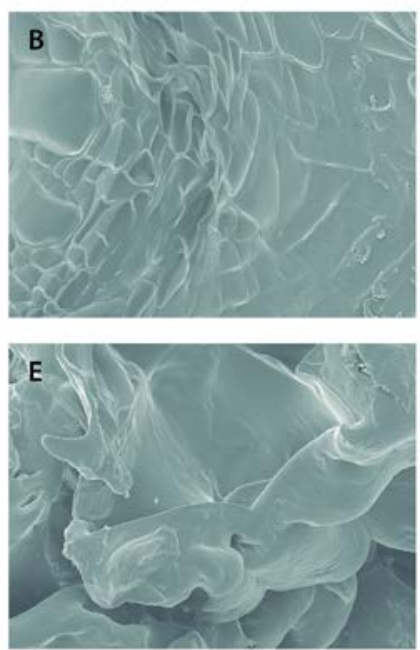

hyal $336 \mathrm{~h}$
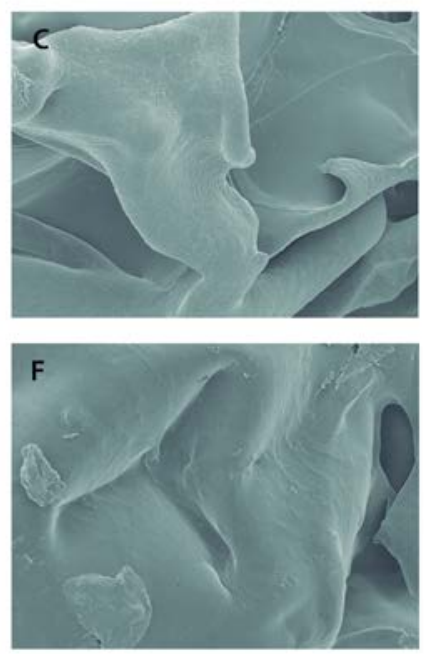
Sup. Figure 2. Elastic modulus for L0 and L0.005 samples at different degradation time points (24, 72, 168 and 336 hours) in water or hyaluronidase-containing medium.

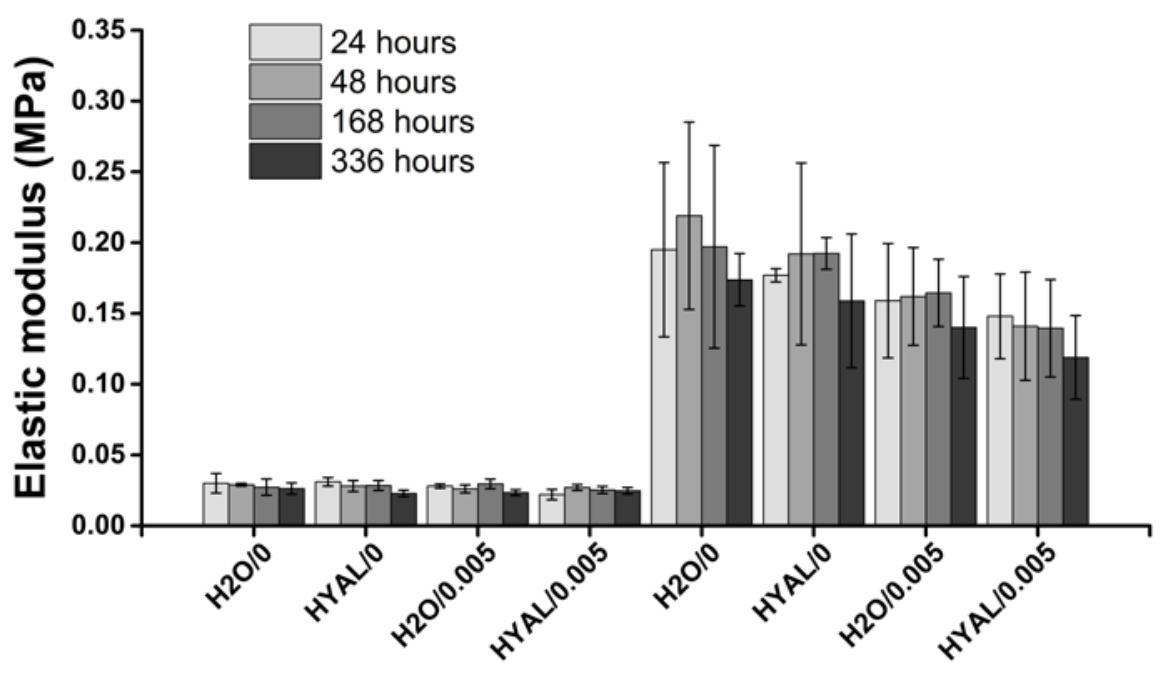

